\title{
Combined VEGF and PDGF treatment reduces secondary degeneration after spinal cord injury
}

Cameron Lutton $^{1 *}$, Yun Wai Young ${ }^{1,2^{*}}$, Richard Williams ${ }^{3}$ Adrian C. B. Meedeniya ${ }^{2}$, Alan Mackay-Sim², Ben Goss ${ }^{3}$

${ }^{1}$ Institute of Health and Biomedical Innovation, Queensland University of Technology, Brisbane, Australia.

${ }^{2}$ National Centre for Adult Stem Cell Research, Eskitis Institute for Cell and Molecular Therapies, Griffith University Brisbane, Australia.

${ }^{3}$ Brisbane Spine Reference Centre, Institute of Health and Biomedical Innovation, Queensland University of Technology, Brisbane, Australia.

*These authors contributed equally to this work 


\section{ABSTRACT}

Trauma to the spinal cord creates an initial physical injury damaging neurons, glia and blood vessels, which then induces a prolonged inflammatory response leading to secondary degeneration of spinal cord tissue and further loss of neurons and glia surrounding the initial site of injury. Angiogenesis is a critical step in tissue repair but in the injured spinal cord angiogenesis fails; blood vessels formed initially, later regress. Stabilizing the angiogenic response is therefore a potential target to improve recovery after for spinal cord injury. Vascular Endothelial Growth Factor (VEGF) can initiate angiogenesis but cannot sustain blood vessel maturation. Platelet Derived Growth Factor (PDGF) can promote blood vessel stability and maturation. We therefore investigated a combined application of VEGF and PDGF as treatment for traumatic spinal cord injury, with the aim to reduce secondary degeneration by promotion of angiogenesis. Immediately after hemi-section of the spinal cord in the rat we delivered VEGF and PDGF and to the injury site. One and three months later the size of the lesion was significantly smaller in the treated group compared to controls and there was significantly reduced gliosis surrounding the lesion. There was no significant effect of the treatment on blood vessel density, although there was a significant reduction in the numbers of macrophages/microglia surrounding the lesion, and a shift in the distribution of morphological and immunological phenotypes of these inflammatory cells. VEGF and PDGF delivered singly exacerbated the secondary degeneration, increasing the size of the lesion cavity. These results demonstrate a novel therapeutic intervention for spinal cord injury and reveal an unanticipated synergy for these growth factors whereby they modulated inflammatory processes and create a microenvironment conducive to axon preservation/sprouting. 


\section{BACKGROUND}

After spinal cord injury, the secondary degenerative changes, gliosis and the formation of inhibitory scars are key factors that limit spontaneous neural regeneration and the effectiveness of regenerative therapies. Traumatic injury disrupts blood vessels and propagates a cyclic breakdown the blood-spinal cord barrier, a continuing inflammatory response and secondary degeneration (Whetstone, et al., 2003). Local angiogenesis is a normal response to traumatic spinal cord injury (Casella, et al., 2002, Loy, et al., 2002) and is a potential target for therapeutic intervention.

Vascular Endothelial Growth Factor (VEGF) is a potent stimulator of angiogenesis and acute delivery of recombinant VEGF (Widenfalk, et al., 2003) or cells overexpressing VEGF (Liu, et al., 2010) can increase angiogenesis outside the epicenter of the lesion after spinal cord contusion injury, rescuing cells locally from ischemic death, reducing tissue loss and improving behavioural outcomes (Kim, et al., 2009, Widenfalk, et al., 2003) . VEGF did not increase angiogenesis at the epicenter of the lesion and without treatment robust vessel growth can occur acutely near the lesion epicenter, only to recede at later time points (Casella, et al., 2002) perhaps because of lack of maturation of newly formed blood vessels (Jain, 2003). Although VEGF can initiate angiogenesis, other factors are usually required for mature vessel formation (Yancopoulos, et al., 2000); one of these is Platelet-derived growth factor (PDGF), which stimulates smooth muscle cell migration promoting blood vessel stability and maturation (Darland and D'Amore, 1999, Hellstrom, et al., 1999). When delivered in combination VEGF and PDGF stimulated a prolonged and robust angiogenic response (Chen, et al., 2007, Hao, et al., 2007, Richardson, et al., 2001). This led us to consider dual growth factor delivery to promote angiogenesis after spinal cord injury in an attempt to reduce secondary degeneration. 
Spinal cord injury elicits a complex spatio-temporal response in the injured tissue, such that a particular growth factor treatment may be useful during a limited period only, complicated by the fact that most growth factors have multiple cell targets. For example, delayed delivery of VEGF can exacerbate the injury by increasing microvascular permeability (Benton and Whittemore, 2003); it can break down the blood-spinal cord barrier and enhance monocyte cell extravasation preceding angiogenesis in the adult brain (Croll, et al., 2004); and it can hasten antigen clearance in the skin (Kataru, et al., 2009). PDGF can enhance early phagocytic activity of neutrophils (Wilson, et al., 1987) and monocytes (Bezuidenhout, et al., 2007) and modulate the activity of T-cells (Daynes, et al., 1991). Although neither growth factor is directly pro-inflammatory, in the presence of a pre-existing inflammatory microenvironment they can be immunomodulatory. Both growth factors target receptor tyrosine kinases expressed on macrophages/microglia and signal through a multitude of pathways including both Janus Kinase/Signal Transducer and Activator of Transcription (JAK/STAT) (Vignais, et al., 1996) and Mitogen Activated Protein (MAP) kinase signaling pathways (Tchaikovski, et al., 2008), which are involved transcription of both pro- and anti-inflammatory responses in macrophages/microglia. When delivered adenovirally to ischemic muscle, VEGF and PDGF in combination resulted in a prolonged increase in vascular perfusion, an effect mediated via recruitment of inflammatory cells rather than directly via angiogenesis (Korpisalo, et al., 2008). This led us to include an analysis of inflammatory cell populations after VEGF and PDGF treatment of the injured spinal cord.

In this study VEGF and PDGF were delivered to the site of injury after hemisection of the rat spinal cord. Two modes of delivery were attempted, acute delivery using a polymer gel and prolonged delivery using an osmotic minipump, with the aim to investigate whether combined growth factor delivery could reduce secondary degeneration after the initial traumatic damage to the spinal cord. 


\section{METHODS}

\section{Preparation of patch and pump implants}

A layered hydrogel patch was made with an impermeable layer and a reservoir layer beneath it to which the growth factors were added in phosphate buffered saline (PBS), as a solvent (Fig. 1a) The reservoir layer consisted of $0.17 \mathrm{ml}$ of $40 \%$ (w/w) Pluronic F127 (Sigma) containing $5 \mu \mathrm{g}$ of recombinant human vascular endothelial cell growth factor-165 (VEGF; Invitrogen) and $5 \mu \mathrm{g}$ of recombinant mouse plateletderived growth factor-BB (PDGF-BB; Invitrogen), a thermally gelling triblock copolymer, $0.14 \mathrm{ml}$ of $50 \%$ (w/w) polyethylene glycol-8000 (PEG-8000; Sigma), $68 \mu 1$ of polyethylene glycol diacrylate (Sigma) and $10 \mu \mathrm{g}$ of the water soluble photoinitiator 2 hydroxy-4'-(2 hydroxyethoxy)-2-propiophenone. This was placed in a $5 \mathrm{~mm}$ x $5 \mathrm{~mm}$ silicone mold and polymerised under high intensity board spectrum ultraviolet light for 10 seconds. The impermeable layer $(0.17 \mathrm{ml} 40 \%$ (w/w) Pluronic F127, $100 \mu 1$ polyethylene glycol diacrylate and $10 \mu \mathrm{g}$ photoinitiator) was placed on top of the already polymerised reservoir layer and polymerised as above. The control gels were synthesised in the same manner but without the addition of growth factors. All gels were stored at $-20 C^{0}$ until needed.

The mini-osmotic pump (Alzet Model 2001, 1 $\mu 1 / \mathrm{hr}$ for 7 days) was weighed prior to loading and then filled with VEGF (Quantikine ${ }^{\circledR}, \mathrm{R} \& \mathrm{D}$ system, Inc) and (Quantikine®, R \& D system, Inc) dissolved in $168 \mu$ l PBS, using a blunt-tipped 27 gauge filling tube provided by the manufacturer (Fig. 1b). For combined treatments the growth factors were $5 \mu \mathrm{g}$ of each (Low Dose) or $15 \mu \mathrm{g}$ of each (High Dose). For single growth factor treatments $15 \mu \mathrm{g}$ of either growth factor was used. The filled pump was weighed and the net weight of the growth factor solution loaded was recorded. A catheter $(15 \mathrm{~cm}$ long) was then connected to the pump and the pump was primed in $0.9 \%$ sterile saline solution at $37{ }^{\circ} \mathrm{C}$ overnight before the pump was implanted. 


\section{Quantification of growth factor release}

Four individual patch implants containing VEGF and PDGF were placed in $1 \mu \mathrm{L}$ PBS at $37 \mathrm{C}^{\circ}$. At different times between 1 and $240 \mathrm{hr}$ later, the $1 \mu \mathrm{L}$ was collected and replaced with $1 \mu \mathrm{L}$ fresh PBS, also at $37 \mathrm{C}^{\circ}$. The concentration of VEGF and PDGF of each time sample were determined using commercially available ELISA kits according to manufacturer's instructions (R\&D systems).

Two osmotic pumps loaded with $5 \mu \mathrm{g}$ VEGF and $5 \mu \mathrm{g}$ PDGF, connected to an intrathecal catheter. The tip of catheter was placed inside a conical tube containing $200 \mu \mathrm{L}$ PBS and the solution was changed every 24 hours. The concentration of growth factors released was quantified using MicroBCA protein assay kits according to the manufacturer's instruction (Thermo Scientific).

\section{Animal surgery}

Adult, male, Wistar rats (Patch experiments: 400g, 20-25weeks old; Pump experiments: 300g, 20-25weeks old) were anesthetised (i.p.; 70 mg/Kg Zoletil 100, Virbac Animal Health; 20mg/Kg Xylazine, Troy Laboratories Pty Ltd) and laminectomies were performed at T10. Lateral hemisections were performed using iridectomy scissors followed by a cut with the tip of a $28 \mathrm{G}$ needle to ensure a complete hemisection.

For the Patch experiments animals were then randomly assigned to survival period (1 month, 3 months) and treatment and control groups (Lesion Control, Gel Control, Growth Factor Treatment; $n=5$ per group at each survival period). In the Treatment and Gel Control groups the implant was placed directly in contact with the dura. The laminectomy was covered by suturing muscle and skin layers above.

For the Pump experiments, an additional hemi-laminectomy was performed at T12 and the catheter was passed under the T11 lamina ipsilateral to the lateral hemisection. The tip of the catheter was carefully placed on top of the lesion and 
sutured to the muscle to keep it in place. Mini-osmotic pumps were implanted in a subcutaneous pouch over the right flank and attached to the implanted catheter. The animals were then randomly assigned to treatment and control groups (LC: Lesion

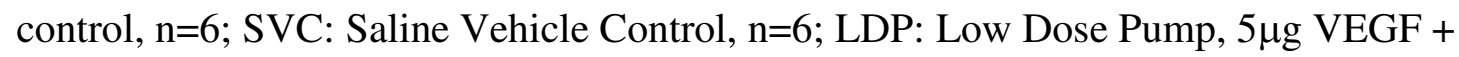

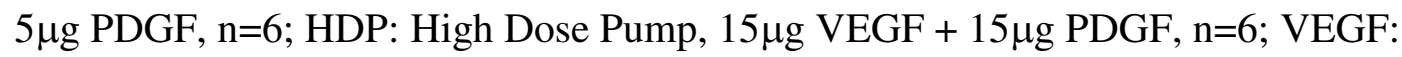
15 $\mu$ g VEGF, $n=3$; PDGF: $15 \mu \mathrm{g}$ PDGF, $\mathrm{n}=3$. The laminectomies were covered by suturing muscle and skin layers above them.

All procedures were approved by the animal ethics committees of the Queensland University of Technology and Griffith University according to the guidelines of the National health and medical Research Council of Australia.

\section{Tissue preparation and immunofluorescence histology}

At 1 or 3 months the animals were sacrificed by anesthetic overdose $(1.5 \mathrm{~mL}$ sodium pentobarbital, i.p.), perfused transcardially with $200 \mathrm{~mL}$ PBS followed by 200 $\mathrm{mL}$ Zamboni's fixative solution, $\mathrm{pH}$ 7.2. The spinal cord in the region of the lesion was dissected out and post-fixed in Zamboni's fixative solution overnight at $4{ }^{\circ} \mathrm{C}$. The spinal cord was then dehydrated (sequential tranfers through 50\%, 70\% and 100\% ethanol solutions, $1 \mathrm{~h}$ each) and then embedded in polyethylene glycol (Bard and Ross, 1986), after which $40 \mu \mathrm{m}$ horizontal sections were cut using a Leica RM2255 rotary microtome (Leica, Germany).

Sections were washed with PBS-Triton (0.1\% Triton X 100 (Merck, Germany) in PBS) for $10 \mathrm{~min}$. Sections were permeabilised in dimethyl sulfoxide for $20 \mathrm{~min}$ and washed three times in PBS-Triton for 15 min. After washing, sections were blocked with $10 \%$ donkey serum (Sigma), $0.1 \%$ Triton X 100 in PBS for 1 hour. antineurofilament 200 (NF200), mouse monoclonal (Chemicon, 1:800); anti-glial fibrillary acidic protein (GFAP), rabbit polyclonal (Dako, Z.0334, 1:2000); antismooth muscle actin, mouse monoclonal (Sigma, A2547, 1:1000); anti- ionized 
calcium binding adaptor molecule 1 (IBA-1), rabbit polyclonal (Wako, 019-19741, 1:2000); Neruron/Glia type 2 antigen (NG2) Rabbit polyclonal (Chemicon 1:500) . Sections were washed three times in PBS-Triton and incubated with Alexa Fluor 594 Donkey anti-mouse IgG (Life Technologies, A-21203, 1:800), Donkey anti-mouse or Alexa Fluor 488 Donkey anti-rabbit IgG (Life Technologies, A-21206, 1:400) overnight at room temperature. Sections were washed three times in PBS-Triton and mounted with Vectorshield mounting media with DAPI (Vector Laboratories).

The specimens were visualised on an Axio Imager Z1 epifluorescence microscope with Apotome, using 5X and 20X Plan-Apochromatic objectives (n.a: 0.30 and 0.75, respectively) and an oil immersion 63X Plan-Apochromatic objective (n.a: 1.40) (Carl Zeiss, Germany). Standard wide-field images and serial optical sections were captured on an AxioCam Mrm camera using AxioVision software. Data was generated, manipulated and analysed using AxioVision (Carl Zeiss, Germany). Standardised exposure times were used for each primary/secondary combination (40ms for GFAP/Alexa Flour 488, 80ms for NF-200/Alexa Flour 594). Figures were compiled in Adobe Photoshop 11.1 (Adobe Systems Incorporated). All quantification of histological measurements were performed blinded to the treatment of the animal.

\section{Quantification of lesion cavity size}

The size of the lesion cavity was measured at the centre of the spinal cord, assessed by the presence of the central canal in these horizontal sections, cross-checked to ensure that this midline section was midway through the stack of serial horizontal sections from dorsal to ventral surface. There was occasionally a discrepancy between these two midline indicators due to minor variations in the orientation of the cord 
during embedding, in which case we used the latter direct measurement of the slice position. The tissue sections were inspected to ensure they were structurally complete, except for the lesion, and stained for GFAP and NF200. After staining, a mosaic of nine adjacent images were taken of the lesion site and surrounding tissue using a 10x objective. These were compiled into a single image using AxioVision 4.7 (Carl Zeiss, Germany) and fluorescent images were complied for both secondary antibodies to define the lesion size by the margin of NF200 and GFAP immunoreactivity. The lesion cavity size was measured by outlining margin of the immunopositive area with the AxioVision 'measure' tool. The measurements of the midline lesion area were averaged over all 5 animals per group. All image analysis was performed with the observer blind to the treatment group.

\section{Quantification of blood vessel density}

Total blood vessel density was determined from tissue sections $140 \mu \mathrm{m}$ ventral to the midline section. These sections were stained for GFAP to determine the lesion margin and Smooth Muscle Actin (SMA) to identify mature blood vessels. Images were taken $200 \mu \mathrm{m}$ from the GFAP defined lesion margin. The area of tissue staining positive for SMA was measured by manually outlining the SMA positive areas using AxioVision 4.7 'measure' tool (Carl Zeiss, Germany) and the density was determined as the percentage of the overall field of view that was outlined as positive for SMA. This was repeated at four locations around the lesion margin for each animal, averaged across animals in each treatment group. Differences in the mean blood vessel density were tested using ANOVA. All image analysis was performed with the observer blind to the treatment group. 


\section{Quantification of gliosis}

Gliosis was quantified as previously described and validated (Romero-Sandoval, et al., 2008). Three dimensional Z-stack mosaic images ( $3 \mu \mathrm{m} \times 5$ slices) were taken at $200 \mu \mathrm{m}$ from the GFAP-defined lesion margin (at least 5 positions per slide) in sections dorsal and ventral to the midline section. GFAP staining was quantified from the pixel intensity in these images. Care was taken to keep all variables constant across all animals. All sections were processed in the same manner for immunofluorescence and the same microscope settings and imaging variables were used for all tissue sections, with exposure time fixed at 40ms. Images were imported into Adobe Photoshop CS2 without altering image settings. A $200 \mu \mathrm{m}$ x $200 \mu \mathrm{m}$ box (i.e. $1 / 4$ of the original image) was chosen from the original images to provide a homogeneous field free of blood vessels, autofluorescence and other artifacts. These cropped images were analysed using MatLab $®$ embedded with Image Processing Toolbox 6.2 with the threshold intensity of 10 ( $>10=$ Bright; $<10$ Dark). Data were presented as percentage of bright pixel in the whole cropped images. All image data was subjected to manual verification and analysed by using Sigma Plot 9.0. All image analysis was performed with the observer blind to the treatment group.

\section{Quantification of microglia/macrophages size distribution}

Three dimensional Z-stack mosaic images were taken at $200 \mu \mathrm{m}$ from the the GFAPdefined lesion margin. Macrophages/microglia were counted was performed using the set of 3D stereological rules described by Williams and Rakic (Williams and Rakic, 1988). Briefly using a 20x objective, five optically defined counting boxes $300 \times 300 \times 25 \mu \mathrm{m}^{3}$ were identified around the lesion perimeter in each of five tissue 
sections taken dorsal and ventral to the midline section of the horizontally sectioned cord ( $\mathrm{n}=25$ fields per animal; 5 animals per group). In each of these fields the total number of IBA1 immuno-positive cells was counted, using DAPI to identify the cell nucleus and IBA1 to identify the cell body, and the size of the cell body at the area of greatest cross-section was measured using the AxioVision 'measure' tool. Cells were only measured if they were entirely contained within the box or intersected the top, rear or right optical planes (Williams and Rakic, 1988).

IBA1-positive cells were classified into classes based on their soma size and morphology, which indicate the activation state of microglia/macrophages (Choo, et al., 2008, Giulian, 1987, Glenn, et al., 1992, Sheng, et al., 1998, Stence, et al., 2001). The cells were classified as: resting, a small ramified appearance, cell body crosssectional area of 20-30 $\mathrm{m}^{2}$; primed / activated, a larger cell (cross-sectional area of $30-150 \mu \mathrm{m}^{2}$ ) with none or few thick processes; phagocytic, very large cell (crosssectional area greater than $150 \mu \mathrm{m}^{2)}$, without processes. All image analysis was performed with the observer blind to the treatment group.

There is recent evidence for a sub-population of IBA1-positive, anti-inflammatory microglia that co-stain with an antibody to NG2 costaining (Smirkin, et al., 2010). Spinal cords were immunostained with antibodies to IBA-1 and NG2 and analysed for co-expression in the same cells using a confocal laser scanning microscope (Leica SP5 CLSM). Typically, Z-stacks were acquired and a cross-sectional profile was assessed using the Leica Microsystems LAS AF software (version 1.8.2 build 1465) to ensure that co- staining occurred at the same location in all three dimensions and truly represented co-localisation of the antibodies. . 


\section{Behavioural Analysis}

In the Patch experiment, functional recovery was evaluated using the Basso-BeattieBresnahan (BBB) open field locomotion scale. This is an observer-rated test which assigns a score between 0 and 21, representing the extremes of no hindlimb movement and normal locomotion respectively (Basso, et al., 1995). This is a well validated scale although with limitations when applied to a hemisection model (Kwon, et al., 2002). Rats were evaluated at different times after lateral hemisection and treatment (1 day, 1 month, 3 months). Behavioural analysis was performed by trained investigators who were blind to the treatment group.

\section{Statistical Analysis}

ANOVA's were performed for lesion size measurements made at individual timepoints and immuno-stains. Post-hoc analysis of significant differences was performed using the Fisher LSD ( $\alpha=0.05, \mathrm{n}=5$ animals in each case). A Kruskal-Wallis ANOVA on ranks was used to compare GFAP density between groups. Post-hoc analysis was performed using Dunn's Method $(\alpha=0.05)$. A minimum of 30 individual tissue samples across animals were used for each group. An ANOVA was used to examine statistical differences between microglia/macrophage populations. Post-hoc analysis of significant differences was performed using the Fisher LSD ( $\alpha=0.05)$. Each measurement was made from a minimum of 17 tissue samples across animals. The BBB scores were analysed by ANOVA. 


\section{RESULTS}

\section{Acute VEGF/PDGF (patch delivery) reduced lesion cavity size}

In vitro testing, which approximates in vivo release, showed that $70 \%$ of the growth factors were delivered from the patch within $48 \mathrm{hrs}$ and that VEGF and PDGF had similar release curves (Fig. 1c). At 1 and 3 months following injury and patch implantation there were dramatic histological differences between treatment and lesion control groups (Fig. 2 a-d). The lesion cavity was defined in two ways; by the margin of axons using NF200 immunoreactivity (Fig. 2f) and by the margin of astrocytes using GFAP immunoreactivity (Fig. 2e). Examination of the treated lesion 1 month after injury showed that the cavity defined by GFAP immunoreactivity was filled with NF200-positive axon fibres and IBA1-positive macrophages/microglia (Fig. 3 a-c). In the control animals at 1 month there were no tissues within the GFAPdefined lesion cavity (Fig d-f). By 3 months in the treatment group the lesion cavity was filled with NF200-positive axons and IBA1-positive macrophages/microglia (Fig $3 \mathrm{~g}-\mathrm{i})$ whereas control lesion cavities remained empty of cells forming a typical posthemisection cystic cavity (Fig. 3 j-1).

In the VEGF/PDGF treated spinal cords, compared to the controls, 1 month after injury there was a significant reduction in lesion cavity size based on NF200 immunoreactivity, with the treated cords measuring approximately one third the size of the controls (Fig. 2f). Three months after injury, the lesion cavity in the treated cords remained the same size as at 1 month, whereas the control lesion cavities increased in size with the treated lesion cavities only one fifth the size of the untreated lesion cavities. (Fig. 2f). When the lesion cavity was defined by the GFAP immunoreactive margin there was no significance difference between treatment and 
control groups at 1 month (Fig. 2e). At 3 months the GFAP-defined lesion cavity was reduced compared to 1 month and similar in size to the NF200-defined cavity (Fig.2 e, f) and significantly smaller than the control lesion cavities which had increased in size from 1 to 3 months (Fig. 2e).

\section{Acute VEGF/PDGF (patch delivery) did not alter blood vessel density}

The blood vessel density in the tissue surrounding the lesion cavity was determined from the SMA-immunoreactivity in the tissue $200 \mu \mathrm{m}$ from the GFAP-defined margin of the lesion cavity (Figure 4). Compared to the uninjured control animals (Fig. 4c), both the Treatment and Control groups (Fig. 4a, b) had significantly higher blood vessel densities but there was no effect of VEGF/PDGF treatment on blood vessel density (Figure 4d).

\section{Acute VEGF/PDGF (patch delivery) reduced gliosis}

Gliosis was quantified by measuring the density of GFAP-immunoreactivity in the tissue $200 \mu \mathrm{m}$ from the GFAP-defined margin of the lesion cavity (Figure 5).

Astrocyte morphology is an indicator of their response to injury: unreactive astrocytes have long, thin branched processes whereas reactive astrocytes have an enlarged cell soma with thicker and shorter processes (Norenberg, 1994, Pekny and Nilsson, 2005). This morphological change is captured by the intensity of GFAP staining, from images collected in a standardized manner (Romero-Sandoval, et al., 2008). Surrounding the lesion cavity in the Control group, the GFAP-immunoreactivity was more extensive and the astrocyte morphology indicative of reactive astrocytes at 1 and 3 months (Fig. 5a, b). In contrast, in the Treatment group astrocyte morphology was indicative of less reactivity at 1 month and unreactive morphology at 3 months 
(Fig. 5c, d). The density of GFAP-immunoreactivity, quantified from automated image pixel counting indicated significantly less GFAP-immunoreactivity in the tissue in the Treatment group at 3 months, compared to the Control group at the same time (Fig. 5e). There was also a significant reduction in GFAP-immunoreactivity in the Treatment group compared at 1 and 3 months (Fig. 5e).

\section{Acute VEGF/PDGF (patch delivery) modulated the macrophage/microglia response to injury}

Resident microglia and macrophages derived from circulating monocytes were investigated by quantifying the IBA1-positive cells in the tissue $200 \mu \mathrm{m}$ from the GFAP-defined margin of the lesion cavity (Figure 6). There were stark differences in the morphology of macrophages/microglia in Treatment and Control tissues. The IBA1-positive cells in the Control group were moderately sized and branched (Fig. $6 a, b)$ whereas those in the Treatment group were large, with few, small processes (Fig. 6c, d). At 1 and 3 months the IBA1-positive cells were larger in Treated animals than in Controls, and generally larger than those observed in undamaged spinal cord at a distance from the lesion cavity. There was a significant increase in the density $\left(\right.$ cells $/ \mathrm{mm}^{3}$ ) of IBA-positive cells in all lesioned animals compared to healthy spinal cord ( $\mathrm{p}<0.05$, data not shown). In the Treatment group at 1 month there was a significant reduction in IBA1-positive cells compared to Controls $(13,798 \pm 1077$ cells per $\mathrm{mm}^{3}$ vs $24,190 \pm 1706$ cells per $\mathrm{mm}^{3}$, respectively, mean $\pm \mathrm{SEM}$; $\left.\mathrm{p}<0.001\right)$. A similar observation was made at 3 months $\left(10,561 \pm 952\right.$ cells per $\mathrm{mm}^{3}$ vs $16,695 \pm$ 440 cells per $\mathrm{mm}^{3}$, respectively, mean $\left.\pm \mathrm{SEM} ; \mathrm{p}<0.001\right)$. In the Treatment group the reduction in IBA1-positive cell number at 3 months compared to 1 month was statistically significant $\mathrm{p}<0.001)$. 
VEGF/PDGF altered the distribution of morphologies of IBA1-positive cells (Fig. 5e,f). One month after injury, there were significantly fewer primed/activated cells in the Treatment group compared to the Control group $(\mathrm{p}<0.001)$ and a significant increase in phagocytic cells, which comprised $29.5 \%$ of the total IBA1-positive population in the Treatment group compared to $8.5 \%$ in the Control $(\mathrm{p}<0.013)$. The proportions of cell types were similar at 3 months, with significantly fewer primed/activated cells in the Treatment group $(\mathrm{p}<0.001)$ and more phagocytic cells $(\mathrm{p}<0.001)$. The proportion of phagocytic cells was $47.5 \%$ in the Treatment group at 3 months, compared with $13.5 \%$ in the control.

\section{Acute VEGF/PDGF (patch delivery) did not improve behavioural recovery}

One day after lateral hemisection all animals had a BBB score of 2 or 3 . One month later the scores had risen, with similar scores obtained by rats in all groups (Lesion Control: $9.6 \pm 2.7$; Gel Control: $10.2 \pm 2$; Treatment: $8.2 \pm 1.8$, Mean \pm SEM). At three months the BBB scores were not significantly different to the scores at one month (Lesion Control: 11.5 \pm 3.3 ; Gel Control: $10.3 \pm 2$; Treatment: $9.6 \pm 0.2$ ).

\section{Prolonged VEGF/PDGF (pump delivery) reduced lesion size, dose dependently} In vitro testing of mini-osmotic pumps loaded with 5 (Low dose) and $15 \mu \mathrm{g}$ (High Dose) VEGF and PDGF showed a sustained release, with 0.7 and $2.1 \mu \mathrm{g}$ the proteins released every 24 hours from Low and high dose pumps respectively (Fig. 1c). More than $95 \%$ VEGF/PDGF was released after 7 days. These values probably reflect release dynamics in vivo because release from these pumps is independent of physiological conditions (Bittner, et al., 2000). 
Combined VEGF/PDGF treatment reduced or eliminated the lesion cavity, assessed by NF200- and GFAP-immunoreactivity (Fig. 7). In contrast, when the growth factors were delivered alone (VEGF, PDGF), the lesion cavity size was very much larger than any of the Controls at 1 and 3 months (SVC, LC). This is strong evidence of a synergy between the action is of the two growth factors in the injured spinal cord. Similar observations were made when the lesion cavity was assessed by NF200immunoreactivity (Fig. 7a-e) or GFAP-immunoreactivity (Fig. 7f-j). The effect of the combined VEGF/PDGF treatment was dose-dependent, with a significantly larger reduction in lesion cavity size at the High Dose compared to Low Dose (Fig. 7e, j; HDP vs LDP). At 3 months the NF200-immunoreactive axons often filled the lesion cavity, making the lesion site difficult to locate (Fig. 7d, inset) and eliminating the lesion cavity altogether (Fig. 7e).

\section{Prolonged VEGF/PDGF (pump delivery) modulated the macrophage/microglia response to injury}

As with the Patch delivery, resident the IBA1-positive cells were quantified in the tissue $200 \mu \mathrm{m}$ from the GFAP-defined margin of the lesion cavity at 1 month (Fig. 8). After combined VEGF/PDGF treatment, in both High (HDP) and Low Dose (LDP) groups, there were significant alterations in the distributions of the morphological classes of IBA1-positive cells (Fig. 8). Overall there was a statistically significant difference between cell size distribution in the Treatment groups and Control groups $(\mathrm{p}<0.001)$. High and Low Dose treatment groups had significantly more phagocytic cells $(\mathrm{p}<0.001)$ with $18.6 \%$ and $19.3 \%$ phagocytic cells (LDP vs HDP, respectively) in the total IBA1-positive cell population compared to $3.6 \%$ in the Control groups 
(LC, SVC) . There were significantly fewer primed/activated cells in the Treatment groups when compared to the Controls. (LDP: p<0.001; HDP: $\mathrm{p}=0.008$ ).

\section{Prolonged VEGF/PDGF (pump delivery) induced a sub-population of IBA-1- positive cells also expressing NG2}

Resident the IBA1-positive cells that co-stained with NG2 were quantified in the tissue $200 \mu \mathrm{m}$ from the GFAP-defined margin of the lesion cavity at 1 month (Fig. 9). In all groups cells were identified that were immunopositive for IBA1-1 (red) and NG2 (green). As previously, IBA-1 staining in the Lesion Control (Fig. 9a, c), VEGF alone (Fig 9 d, f), and PDGF alone (Fig. 9h, j) revealed stellate macrophages /microglia with thick processes, indicative of an active morphology. In contrast, the microglia/macrophages in the High Dose VEGF/PDGF group were large, rounded, without processes, indicative of a phagocytic morphology (Fig 9 k, m). Moreover in the High Dose VEGF/PDGF group, there were a large number of IBA1-positive cells that co-expressed NG2 (Fig. 9 1, m). The Control group showed the least evidence of NG2 staining (Fig. 9 b, c), the NG2 staining in the PDGF group was sparse (Fig. 9 i, j), and in the VEGF group there were a few small, isolated NG2-positive cells (Fig. 9 e, f) that resemble to oligodendrocyte precursors (Jones, et al., 2002). These latter cells were not IBA1-positive. 


\section{DISCUSSION}

We show here that after acute traumatic spinal cord injury VEGF and PDGF, delivered in combination, reduced the lesion size and reduced gliosis around the injury at 1 month and prevented further increases at 3 months, compared to control animals. In stark contrast, the growth factors administered singly were similar or worse than controls. VEGF/PDGF treatment did not promote angiogenesis but it significantly modulated the inflammatory cell response, inducing a population of microglia/macrophages that were immuno-positive for NG2, and altering the distributions of microglia/macrophages classified as "resting", "activated/primed" and "phagocytic". Growth factor treatment significantly decreased the numbers of activated/primed" cells and increased the numbers of "phagocytic" cells at 1 and 3 months in the injured tissue. These same effects were seen after acute treatment delivered by hydrogel patch and by sustained treatment delivered by osmotic minipump, with the latter experiments demonstrating a dose-dependency of the effects of growth factor treatment. VEGF/PDGF treatment also promoted robust axon growth into the lesion cavity.

There has been mixed success in prior experiments using VEGF to treat spinal cord injury. It is reported to promote angiogenesis, gliogenesis, neuronal survival and tissue sparing; to decrease secondary degeneration; to improve functional outcomes (Kim, et al., 2009, Liu, et al., 2010, Storkebaum, et al., 2004, Sundberg, et al., 2011, Widenfalk, et al., 2003); and to induce regeneration of corticospinal tract axons (Facchiano, et al., 2002). Others have observed no effect of VEGF treatment (van Neerven, et al., 2010), or worse outcomes (Benton and Whittemore, 2003). This complexity is likely due to the methodological differences in the types of spinal cord 
injuries and the timing of VEGF delivery interacting with the pleiotropic nature of the growth factor and the varying microenvironments that occur in the different times after injury. In our experiments, VEGF treatment alone was detrimental leading to an increase in lesion size, as reported previously (Benton and Whittemore, 2003), rather than the decrease observed when VEGF was delivery in combination with PDGF.

PDGF has not been used previously after spinal cord injury. It was not neuroprotective effect in an ischemic model of brain trauma (Iihara, et al., 1997) and its absence did not influence the astroglial and angiogenic response in adult brain after neuron specific genetic ablation of PDGF (Enge, et al., 2003). Endogenous expression of PDGF-B and its receptor PDGFR $\beta$ expression increased in rhesus monkey spinal cord after hemisection injury and administration of an antibody to PDGF-B reduced recovery of hindlimb function in this model (Xiyang, et al., 2009). In our experiments, PDGF-B treatment alone was detrimental leading to an increase in lesion size, even larger than after VEGF delivery.

The occurrence of secondary degeneration can be inferred when the size of the lesion increases with time after the physical damage of the initial traumatic injury. VEGF/PDGF combined treatment reduced or eliminated secondary degeneration, as evidenced by the dose-dependent reduction in lesion size at 1 and 3 months after injury. This was accompanied by a reduction in gliosis around the lesion site and the growth of axons into the lesion cavity in the treated animals. The cellular mechanisms responsible for these treatment effects are unclear. Unexpectedly, there was no measureable angiogenic stimulation by the combined treatment, ruling out this hypothesized mechanism. On the other hand, we did observe a robust effect of 
treatment on the inflammatory response, as evidenced by the changes in distributions of microglia/macrophages subtypes within the injured tissues. Treatment induced a significant rise in the population of macrophage/microglia in our 'phagocytic' classification, a concomitant reduction in the population of 'activated/primed' macrophage/microglia, and an increase in the numbers of NG2-positive macrophage/microglia. Without a time-series it is hard to interpret whether these differences reflect a progression of states from pro- to anti-inflammatory macrophage/microglia states or recruitment of different subpopulations to the lesion.

Without further investigation we cannot be certain of the roles of macrophage/microglia populations in reducing secondary degeneration changes but the observations suggest that the treated spinal cords are in a less inflammatory state than controls, with a significant reduction in the numbers of macrophages/microglia as well as the change in distributions of morphologies. Most striking was the appearance of IBA+/NG2+ macrophages, present only in the VEGF/PDGF combination group. Such cells have been observed previously in the 7 days following traumatic spinal cord injury, decreasing to very low levels by 28 days (Jones, et al., 2002). Similar IBA+/NG2+ macrophages may be neuroprotective as they express several mRNAs encoding for growth factors (IGF, FGF2, BMP-2,-4,-7, and HGF) and they ameliorate the effects of ischemia when transplanted into the adult brain (Smirkin, et al., 2010). We show here for the first time a treatment that induces a population of IBA-/NG2-positive macrophages/microglia at extended times after the injury.

Combined growth factor treatment failed to improve the functional outcome as measured by the BBB open-field locomotor scale. This method has been used 
previously after spinal cord hemisection injury. As we observed, there is normally a spontaneous recovery of hindlimb function in rats after hemisection due to recovery from spinal shock, plasticity of spinal circuits and short distance axonal sprouting that can reconnect motor neurons in a path that circumvents the defect (Bareyre, et al., 2004, Hiersemenzel, et al., 2000, Weidner, et al., 2001) and some experimental treatments promote behavioural improvements by augmenting these effects (Kwon, et al., 2010). In our experiments there was no behavioural improvement above the spontaneous recovery, despite the reduced secondary degeneration and the obvious stimulation of axon growth into the lesion cavity. Further investigation is required. We did not test behaviour in the animals receiving the higher dose of VEGF/PDGF, i.e. those with the greatest reduction in lesion cavity size and gliosis, and there may be a threshold in these that is permissive for anatomical changes leading to functional recovery. Neither do we know at this stage the fate of ascending sensory and descending motor axons that would mediate behavioural recovery. We are currently undertaking detailed behavioural studies after VEGF/PDGF treatment of contused spinal cord injuries, a more appropriate model for assessing behavioural outcomes relevant to human injuries.

\section{Conclusions}

In summary, this study has demonstrated for the first time a treatment that can reduce or eliminate secondary degeneration after traumatic injured to the spinal cord, namely, acute or sustained treatment with a combination of VEGF/PDGF. Surprisingly, treatment with the single growth factors exacerbated the secondary degeneration, indicating a previously unknown iteraction and potentiation between these growth factors after injury. Although known for their angiogenic effects, the elimination of 
secondary degeneration by VEGF/PDGF combined treatment was not due to their promotion of angiogenesis in the injured spinal cord. The mechanism for this effect cannot be determined but there is ample evidence for alterations in the populations of macrophages/microglia within the injured tissues that suggest a pathway for future investigation of VEGF/PDGF action.

\section{ACKNOWLEDGEMENTS}

The authors are grateful to The Institute of Health and Biomedical Innovation, Queensland University of Technology, for seed funding, the National Centre for Adult Stem Cell Research, Griffith University, and the Australian Department of Health and Ageing for a Ph.D stipend for Y-W.Y. We appreciate the assistance of the animal house staff at the Medical Engineering Research Facility (Queensland University of Technology). B.G. gratefully acknowledges the Queensland Government for a Smart State Fellowship and the Queensland Orthopaedic Research Trust for ongoing support.

Competing Interests: None of the authors have competing interests in this study. 


\section{REFERENCES}

Bard, J.B. and Ross, A.S. (1986). Improved method for making high-affinity sections of soft tissue embedded in polyethylene glycol (PEG): its use in screening monoclonal antibodies. The journal of histochemistry and cytochemistry : official journal of the Histochemistry Society. 34, 1237-1241.

Bareyre, F.M., Kerschensteiner, M., Raineteau, O., Mettenleiter, T.C., Weinmann, O. and Schwab, M.E. (2004). The injured spinal cord spontaneously forms a new intraspinal circuit in adult rats. Nat. Neurosci. 7, 269-277.

Basso, M., Beattie, M. and Bresnahan, J. (1995). A Sensitive and Reliable Locomotor Rating Scale for Open Field Testing in Rats. J. Neurotrauma. 12, 1-21.

Benton, R. and Whittemore, S. (2003). VEGF165 Therapy Exacerbates Secondary Damage following Spinal Cord Injury. Neurochem. Res. 28, 1693-1703.

Bezuidenhout, L., Bracher, M., Davison, G., Zilla, P. and Davies, N. (2007). Ang-2 and PDGF-BB cooperatively stimulate human peripheral blood monocyte fibrinolysis. J. Leukoc. Biol. 81, 1496-1503.

Bittner, B., Thelly, T., Isel, H. and Mountfield, R.J. (2000). The impact of co-solvents and the composition of experimental formulations on the pump rate of the ALZET osmotic pump. Int. J. Pharm. 205, 195-198.

Casella, G.T., Marcillo, A., Bunge, M.B. and Wood, P.M. (2002). New vascular tissue rapidly replaces neural parenchyma and vessels destroyed by a contusion injury to the rat spinal cord. Exp. Neurol. 173, 63-76.

Chen, R.R., Silva, E.A., Yuen, W.W. and Mooney, D.J. (2007). Spatio-temporal VEGF and PDGF delivery patterns blood vessel formation and maturation. Pharm. Res. 24, 258-264.

Croll, S., Ransohoff, R., Cai, N., Zhang, Q., Martin, F., Wei, T., Kasselman, L., Kintner, J., Murphy, A., Yancopoulos, G. and Wiegand, S. (2004). VEGFmediated inflammation precedes angiogenesis in adult brain. Exp. Neurol. 187, 388- 402.

Darland, D.C. and D'Amore, P.A. (1999). Blood vessel maturation: vascular development comes of age. J. Clin. Invest. 103, 157-158.

Daynes, R., Dowdl, T. and Araneo, B. (1991). Platelet-derived Growth Factor Is a Potent Biologic Response Modifier of T Cells. J. Exp. Med. 174, 1323-1333.

Enge, M., Wilhelmsson, U., Abramsson, A., Stakeberg, J., Kühn, R., Betsholtz, C. and Pekny, M. (2003). Neuron-Specific Ablation of PDGF-B Is Compatible with Normal Central Nervous System Development and Astroglial Response to Injury. Neurochem. Res. 28, 271-279.

Facchiano, F., Fernandez, E., Mancarella, S., Maira, G., Miscusi, M., D'Arcangelo, D., Cimino-Reale, G., Falchetti, M.L., Capogrossi, M.C. and Pallini, R. (2002). Promotion of regeneration of corticospinal tract axons in rats with recombinant vascular endothelial growth factor alone and combined with adenovirus coding for this factor. J. Neurosurg. 97.

Hao, X., Silva, E.A., Mansson-Broberg, A., Grinnemo, K.H., Siddiqui, A.J., Dellgren, G., Wardell, E., Brodin, L.A., Mooney, D.J. and Sylven, C. (2007).

Angiogenic effects of sequential release of VEGF-A165 and PDGF-BB with alginate hydrogels after myocardial infarction. Cardiovasc. Res. 75, 178-185. 
Hellstrom, M., Kalen, M., Lindahl, P., Abramsson, A. and Betsholtz, C. (1999). Role of PDGF-B and PDGFR-beta in recruitment of vascular smooth muscle cells and pericytes during embryonic blood vessel formation in the mouse. Development. 126, 3047-3055.

Hiersemenzel, L.-P., Curt, A. and Dietz, V. (2000). From spinal shock to spasticity: Neuronal adaptations to a spinal cord injury Neurology. 54, 1574-1582.

Iihara, K., Hashimoto, N., Tsukahara, T., Sakata, M., and, H.Y. and Taniguchi, T. (1997). Platelet-Derived Growth Factor-BB, But Not -AA, Prevents Delayed Neuronal Death After Forebrain Ischemia in Rats. J. Cereb. Blood Flow Metab. 17, 1097-1106.

Jain, R.K. (2003). Molecular regulation of vessel maturation. Nat. Med. 9, 685-693.

Jones, L.L., Yamaguchi, Y., Stallcup, W.B. and Tuszynski, M.H. (2002). NG2 is a major chondroitin sulfate proteoglycan produced after spinal cord injury and is expressed by macrophages and oligodendrocyte progenitors. J. Neurosci. 22, 2792-2803.

Kataru, R., Jung, K., Jang, C., Yang, H., Schwendener, R., Baik, R., Han, S., Alitalo, K. and Koh, G. (2009). Critical role of CD11b macrophages and VEGF in inflammatory lymphangiogenesis, antigen clearance, and inflammation resolution. Blood. 113, 5650-5659.

Kim, H., Hwang, D., Lee, J., Kim, S. and Kim, B. (2009). Ex Vivo VEGF Delivery by Neural Stem Cells Enhances Proliferation of Glial Progenitors, Angiogenesis, and Tissue Sparing after Spinal Cord Injury. PLoS Med. 4, e4987.

Korpisalo, P., Karvinen, H., Rissanen, T.T., Kilpijoki, J., Marjomaki, V., Baluk, P., McDonald, D.M., Cao, Y., Eriksson, U., Alitalo, K. and Yla-Herttuala, S. (2008). Vascular endothelial growth factor-A and platelet-derived growth factor-B combination gene therapy prolongs angiogenic effects via recruitment of interstitial mononuclear cells and paracrine effects rather than improved pericyte coverage of angiogenic vessels. Circ. Res. 103, 1092-1099.

Kwon, B.K., Okon, E., Hillyer, J., Mann, C., Baptiste, D., Weaver, L., Fehlings, M. and Tetzlaff, W. (2010). A Systematic Review of Non-Invasive Pharmacologic Neuroprotective Treatments for Acute Spinal Cord Injury. J. Neurotrauma. 27, 1-44.

Kwon, B.K., Oxland, T.R. and Tetzlaff, W. (2002). Animal models used in spinal cord regeneration research. Spine. 27, 1504-1510.

Liu, Y., Figley, S., Spratt, S.K., Leem G, Andom D, Suroskym R and Fehlings M.G. (2010). An engineered transcription factor which activates VEGF-A enhances recovery after spinal cord injury. Neurobiol. Dis. 37, 384-393.

Loy, D.N., Crawford, C.H., Darnall, J.B., Burke, D.A., Onifer, S.M. and Whittemore, S.R. (2002). Temporal progression of angiogenesis and basal lamina deposition after contusive spinal cord injury in the adult rat. J. Comp. Neurol. 445, 308-324.

Norenberg, M.D. (1994). Astrocyte responses to CNS injury. J. Neuropathol. Exp. Neurol. 53, 213-220.

Pekny, M. and Nilsson, M. (2005). Astrocyte activation and reactive gliosis. Glia. 50, 427-434.

Richardson, T.P., Peters, M.C., Ennett, A.B. and Mooney, D.J. (2001). Polymeric system for dual growth factor delivery. Nat. Biotechnol. 19, 1029-1034. 
Romero-Sandoval, A., Chai, N., Nutile-McMenemy, N. and Deleo, J.A. (2008). A comparison of spinal Iba1 and GFAP expression in rodent models of acute and chronic pain. Brain Res. 1219, 116-126.

Smirkin, A., Matsumoto, H., Takahashi, H., Inoue, A., Tagawa, M., Ohue, S., Watanabe, H., Yano, H., Kumon, Y., Ohnishi, T. and Tanaka, J. (2010). Iba1(+)/NG2(+) macrophage-like cells expressing a variety of neuroprotective factors ameliorate ischemic damage of the brain. J. Cereb. Blood Flow Metab. 30, 603-615.

Storkebaum, E., Lambrechts, D. and Carmeliet, P. (2004). VEGF: once regarded as a specific angiogenic factor, now implicated in neuroprotection. Bioessays. 26, 943-954.

Sundberg, L.M., Herrera, J.J. and Narayana, P.A. (2011). Effect of Vascular Endothelial Growth Factor Treatment in Experimental Traumatic Spinal Cord Injury: In Vivo Longitudinal Assessment. J. Neurotrauma.

Tchaikovski, V., Fellbrich, G. and Waltenberger, J. (2008). The Molecular Basis of VEGFR-1 Signal Transduction Pathways in Primary Human Monocytes. Arterioscler. Thromb. Vasc. Biol. 28, 322-328.

van Neerven, S., Joosten, E.A., Brook, G.A., Lambert, C.A., Mey, J., Weis, J., Marcus, M.A., Steinbusch, H.W., van Kleef, M., Patijn, J. and Deumens, R. (2010). Repetitive intrathecal VEGF(165) treatment has limited therapeutic effects after spinal cord injury in the rat. J. Neurotrauma. 27, 1781-1791.

Vignais, M.L., Sadowski, H.B., Watling, D., Rogers, N.C. and Gilman, M. (1996). Platelet-Derived Growth Factor Induces Phosphorylation of Multiple JAK Family Kinases and STAT Proteins. Mol. Cell. Biol. 16, 1759-1769.

Weidner, N., Ner, A., Salimi, N. and Tuszynski, M. (2001). Spontaneous corticospinal axonal plasticity and functional recovery after adult central nervous system injury. Proc. Natl. Acad. Sci. U. S. A. 98 3513-3518.

Whetstone, W.D., Hsu, J.Y., Eisenberg, M., Werb, Z. and Noble-Haeusslein, L.J. (2003). Blood-spinal cord barrier after spinal cord injury: relation to revascularization and wound healing. J. Neurosci. Res. 74, 227-239.

Widenfalk, J., Lipson, A., Jubran, M., Hofstetter, C., Ebendal, T., Cao, Y. and Olson, L. (2003). Vascular endothelial growth factor improves functional outcome and decreases secondary degeneration in experimental spinal cord contusion injury. Neuroscience. 120, 951-960.

Williams, R.W. and Rakic, P. (1988). Three-dimensional counting: an accurate and direct method to estimate numbers of cells in sectioned material. J. Comp. Neurol. 278, 344-352.

Wilson, E., Laster, S., Gooding, L. and Lambeth, J. (1987). Platelet-derived growth factor stimulates phagocytosis and blocks agonist-induced activation of the neutrophil oxidative burst: A possible cellular mechanism to protect against oxygen radical damage. Proc. Natl. Acad. Sci. U. S. A. 84, 2213-2217.

Xiyang, Y.-B., Liu, S., Liu, J., Hao, C.-G., Wang, Z.-J., Ni, W., Wang, X.-Y. and Wang, T.-H. (2009). Roles of Platelet-Derived Growth Factor-B Expression in the Ventral Horn and Motor Cortex in the Spinal Cord-Hemisected Rhesus Monkey. J. Neurotrauma. 26.

Yancopoulos, G.D., Davis, S., Gale, N.W., Rudge, J.S., Wiegand, S.J. and Holash, J. (2000). Vascular-specific growth factors and blood vessel formation. Nature. 407, 242-248. 
a

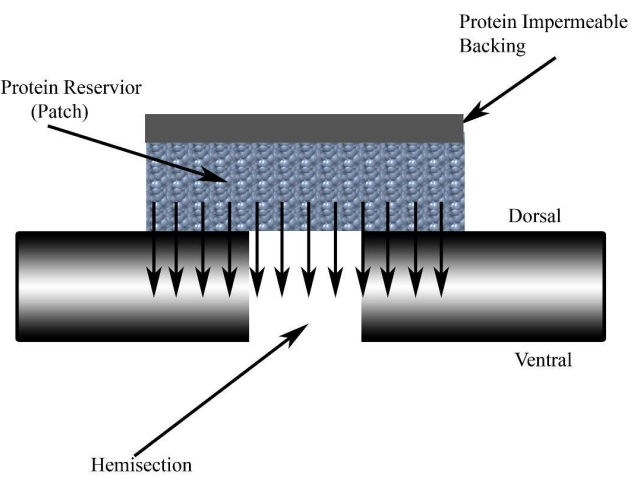

b
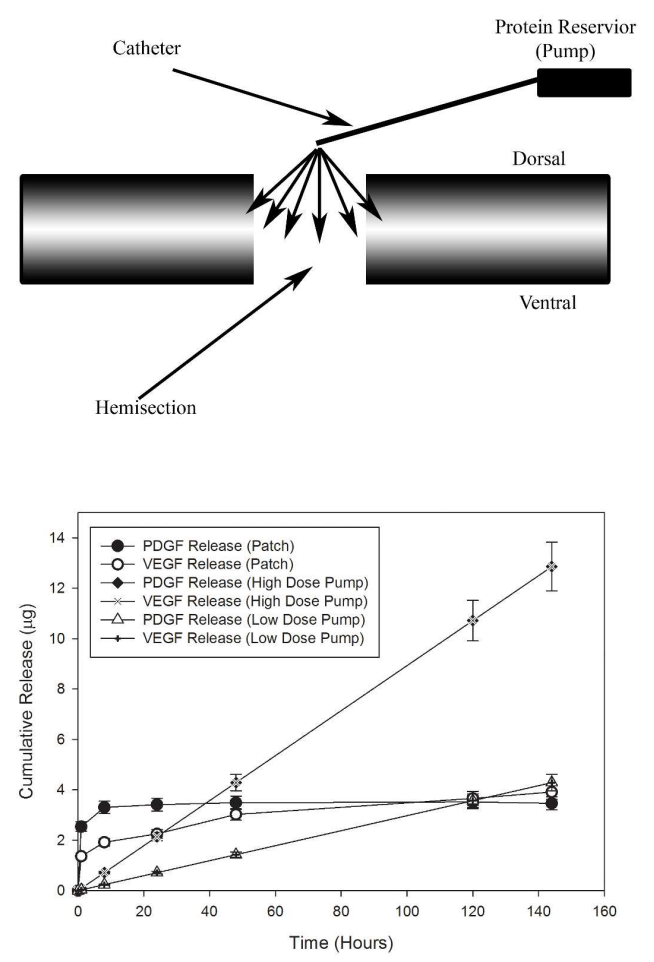

Figure 1: Patch and pump, implantation and delivery profiles a) Schematic of the double layer hydrogel patch created to deliver the growth factors to the injury site b) Schematic of Alzet $\circledast$ miniosmotic pump to deliver the growth factors to the injury site c) Plot of the individual delivery profiles of VEGF and PDGF from a hydrogel patch, low dose pump and high dose pump. (mean \pm standard deviation). The three delivery profiles differed with the hydrogel patch delivering the majority of its payload within the first 48 hours, whilst the two pumps showed a sustained delivery over the 7 day period. $314 \times 445 \mathrm{~mm}(300 \times 300 \mathrm{DPI})$ 

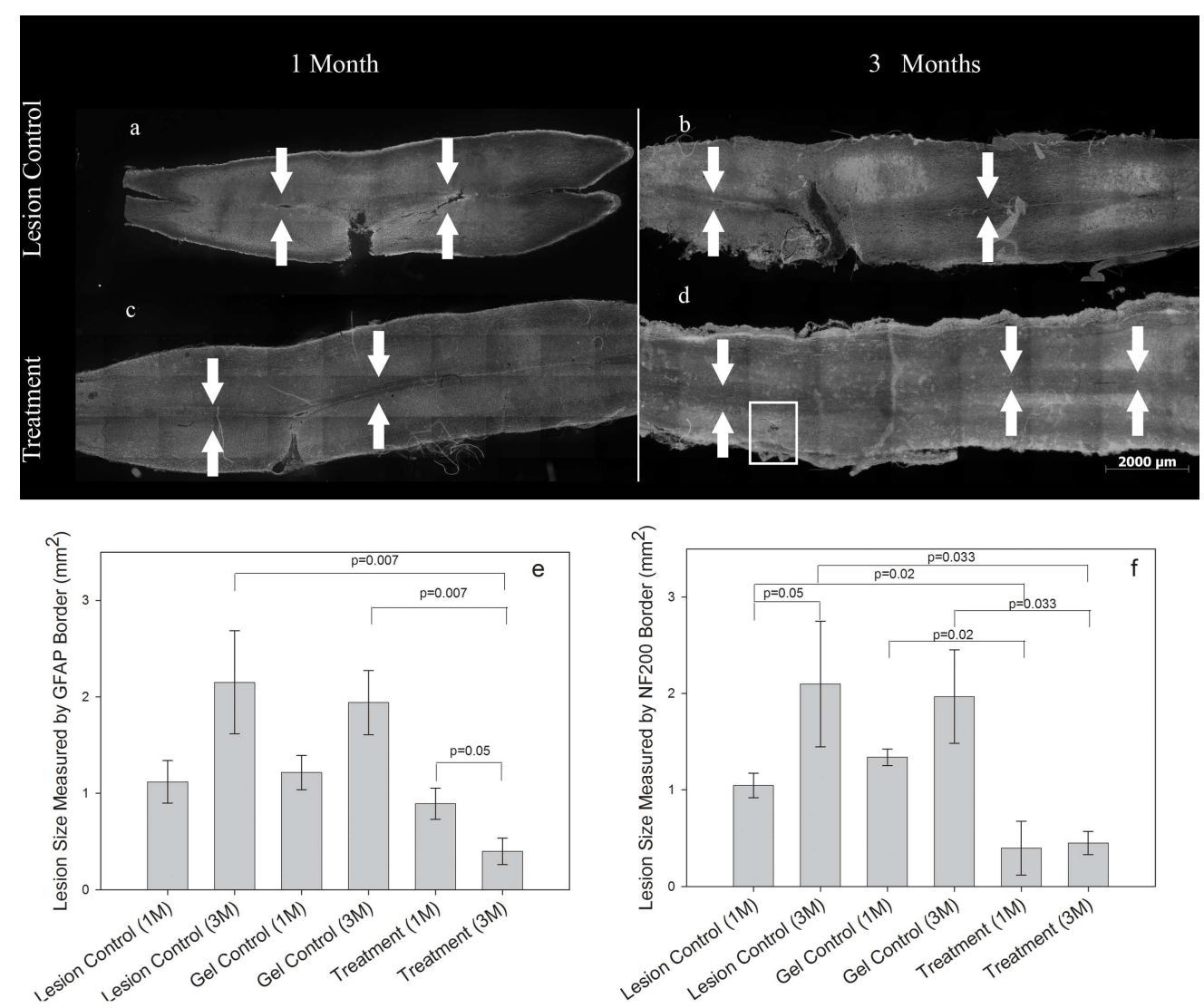

Figure 2: Treatment with VEGF and PDGF delivered from a patch reduced the size of spinal cord lesion. Combined NF200/GFAP black and white images of whole spinal cords harvested at 1 and 3 months post lesion. (a) 1 month lesion control, (b) 3 month lesion control, (c) 1 month treatment and (d) 3 month treatment. Measurement of the size of the spinal cord lesion at one and three months post-injury with the lesion margin defined by (e) glial acidic fibrillary protein (GFAP) margin and (f) neurofilament-200 (NF200) (mean \pm standard deviation) Arrows indicate central canal to show that they are true midline sections. $635 \times 541 \mathrm{~mm}(100 \times 100 \mathrm{DPI})$ 
Figure 3: Treated lesions were filled with IBA-1 and NF200. Combined growth factor treatment resulted in the lesions being filled with IBA1 and NF200 by one month $(a-c)$ compared to untreated controls (d-f). Three months after injury, treated cords showed significant reduction in the lesion size ( $g-i)$ whilst untreated lesions $(j-l)$ have increased in size and remain empty of inflammatory, nueral and glial tissue and have the typical appearance of chronic hemisection injury $199 \times 266 \mathrm{~mm}(300 \times 300 \mathrm{DPI})$

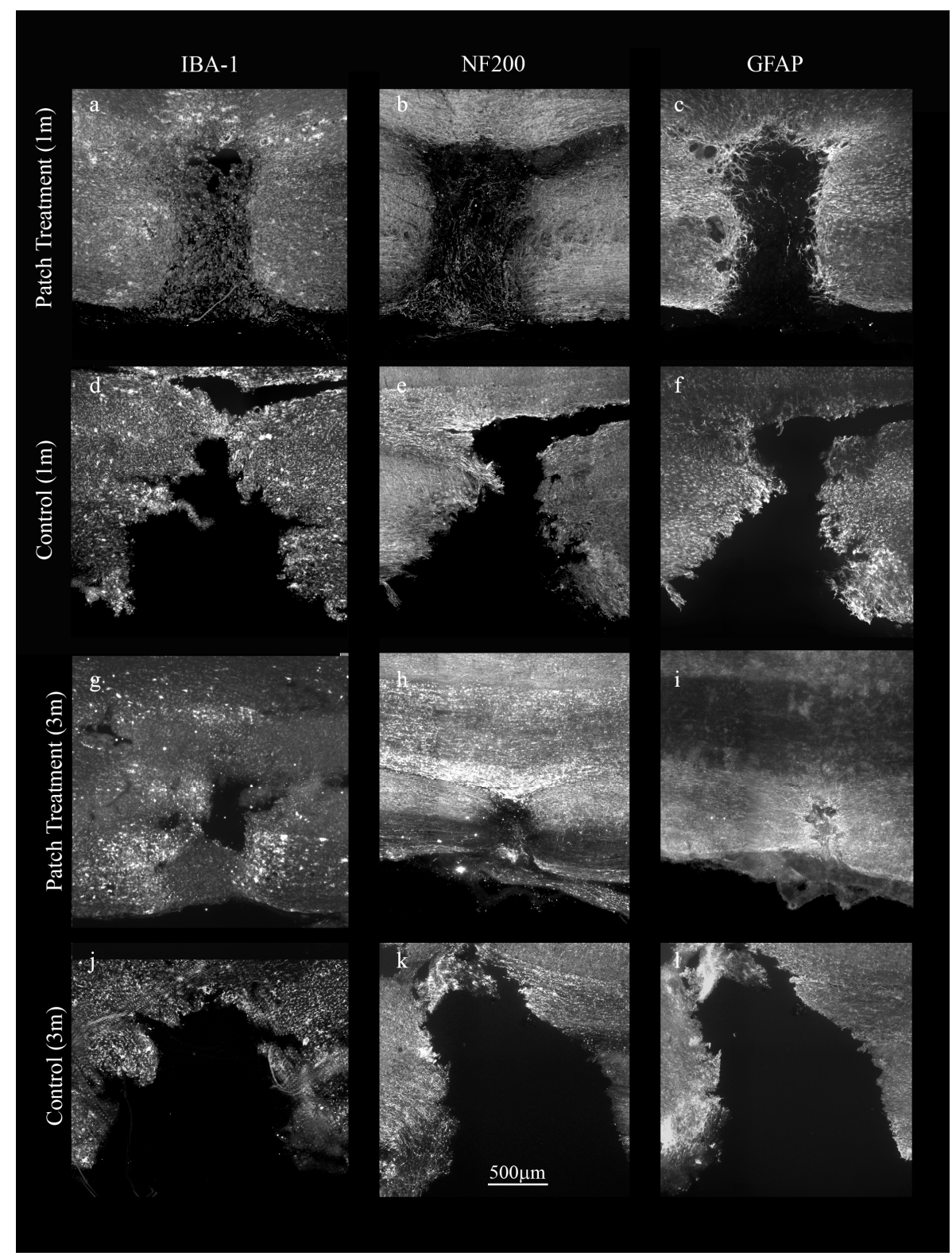



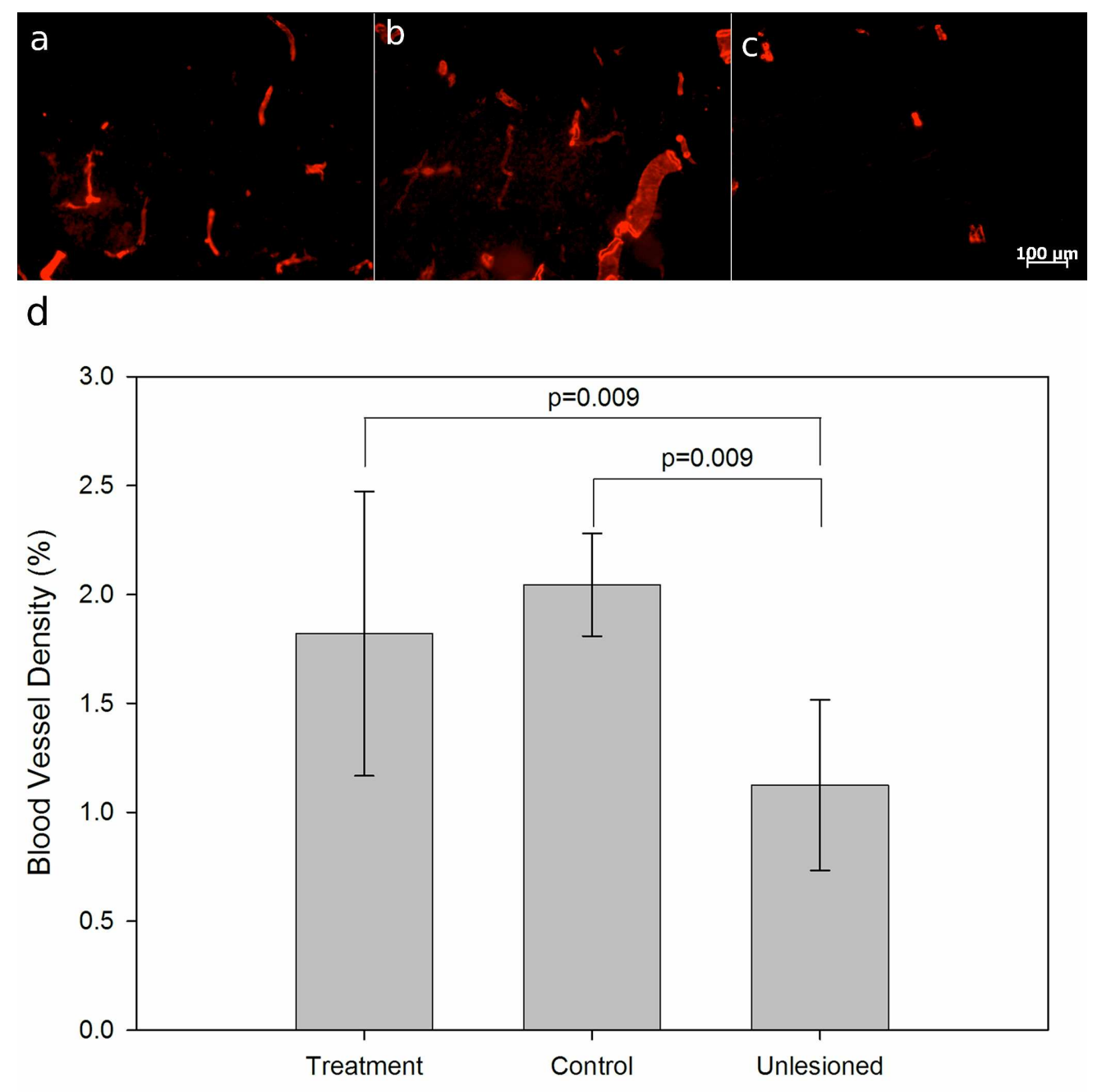

Figure 4 delivery of a combination of VEGF/PDGF did not enhance angiogenesis over the endogeneous angiogenic response to injury. Representative immunoflourescence images showing smooth muscle actin (SMA) staining for (a) Treatment (b) Control and (c) unlesioned spinal cords shows clear tubular structures. The quantification of blood vessel density (d) showed that there were no significant differences between treatment and control, but that both were elevated compared to uninjured tissue $1056 \times 1081 \mathrm{~mm}(100 \times 100 \mathrm{DPI})$ 

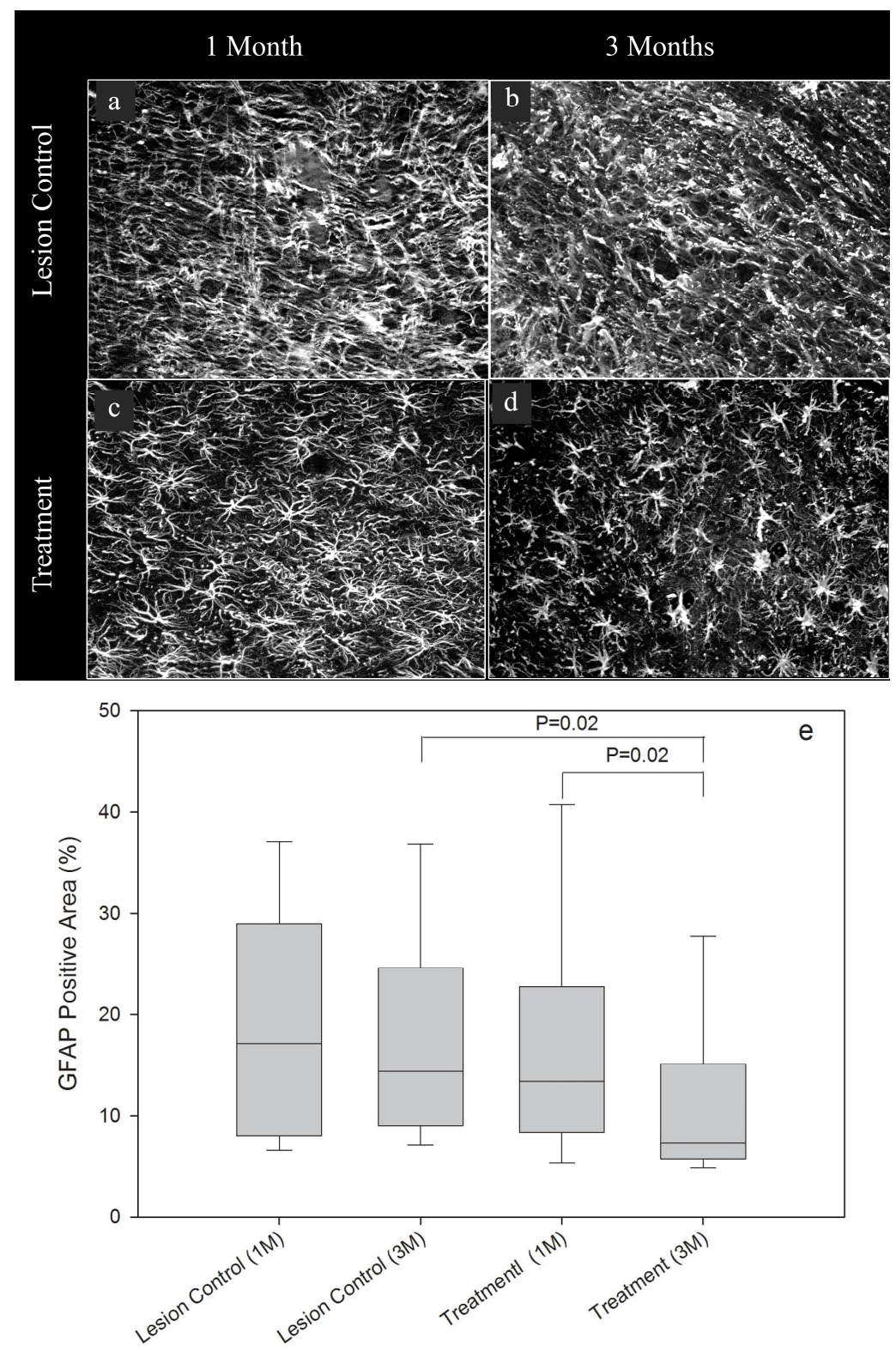

Figure 5: Treatment with VEGF and PDGF reduced astrogliosis. GFAP immunofluorescence images taken $200 \mu \mathrm{m}$ from the lesion, (a) 1 month (b) 3 month lesion control (c) 1 month (d) 3 months treatment group show clear differences in astrocyte morphology and GFAP staining density. This was quantified using image analysis (e) and at 3 months the area occupied by GFAP-positive cells in the treatment group was significantly smaller than in 3 month controls and the 1 month treatment. (whiskers $95 \% \mathrm{CI}$ ) $1072 \times 1645 \mathrm{~mm}(72 \times 72 \mathrm{DPI})$ 

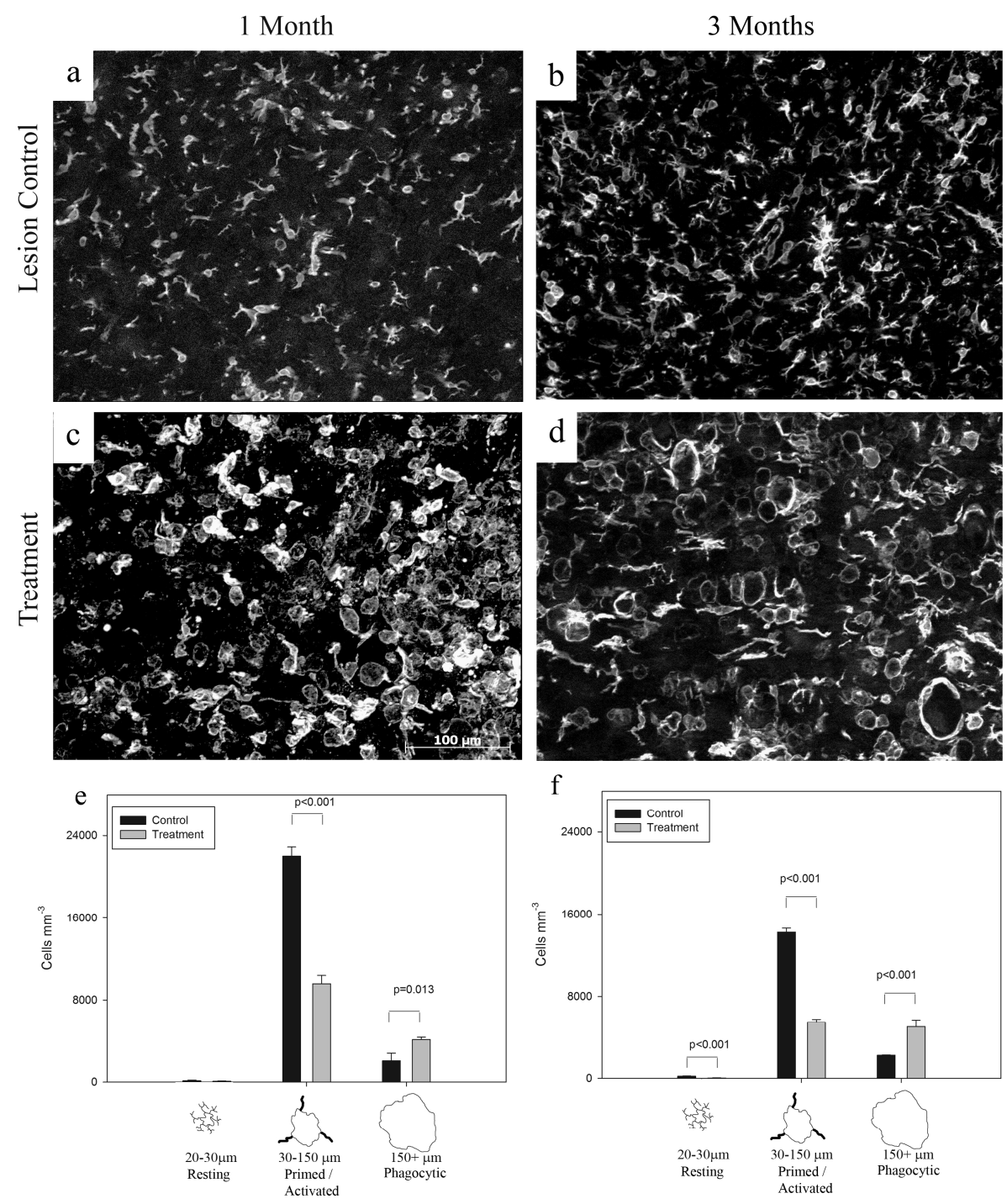

Figure 6: Treatment with VEGF and PDGF altered the inflammatory cell population. Representative IBA-1 immunofluorescence images $200 \mu \mathrm{m}$ from the lesion (a) 1 Month lesion control (b) 3 month lesion control (c) 1 month treatment (d) 3 month treatment show an altered morphology of macrophage/microglia. These cells were classified based on cell body area and counted. The density of IBA1-positive cells classes surrounding the lesion at (a) 1 month and (b) 3 months was significantly different between treatment and control groups. $823 \times 954 \mathrm{~mm}(100 \times 100 \mathrm{DPI})$ 
Figure 7: Treatment with VEGF and PDGF delivered from a mini-osmotic pump reduced the size of spinal cord lesion. Whole spinal cords harvested at 1 month post lesion stained for NF200 or GFAP $(a, f)$ lesion control $(b, g)$ VEGF alone $(c, h)$ PDGF alone $(d, i)$ VEGF/PDGFLesions were quantified around (e) NF200 margin and (j) GFAP margin. (mean \pm standard deviation). This shows a significant increase in the size of the lesion when either VEGF or PDGF is delivered alone and a significant reduction in lesion size when the growth factors were delivered together. $209 \times 212 \mathrm{~mm}(300 \times 300 \mathrm{DPI})$ 


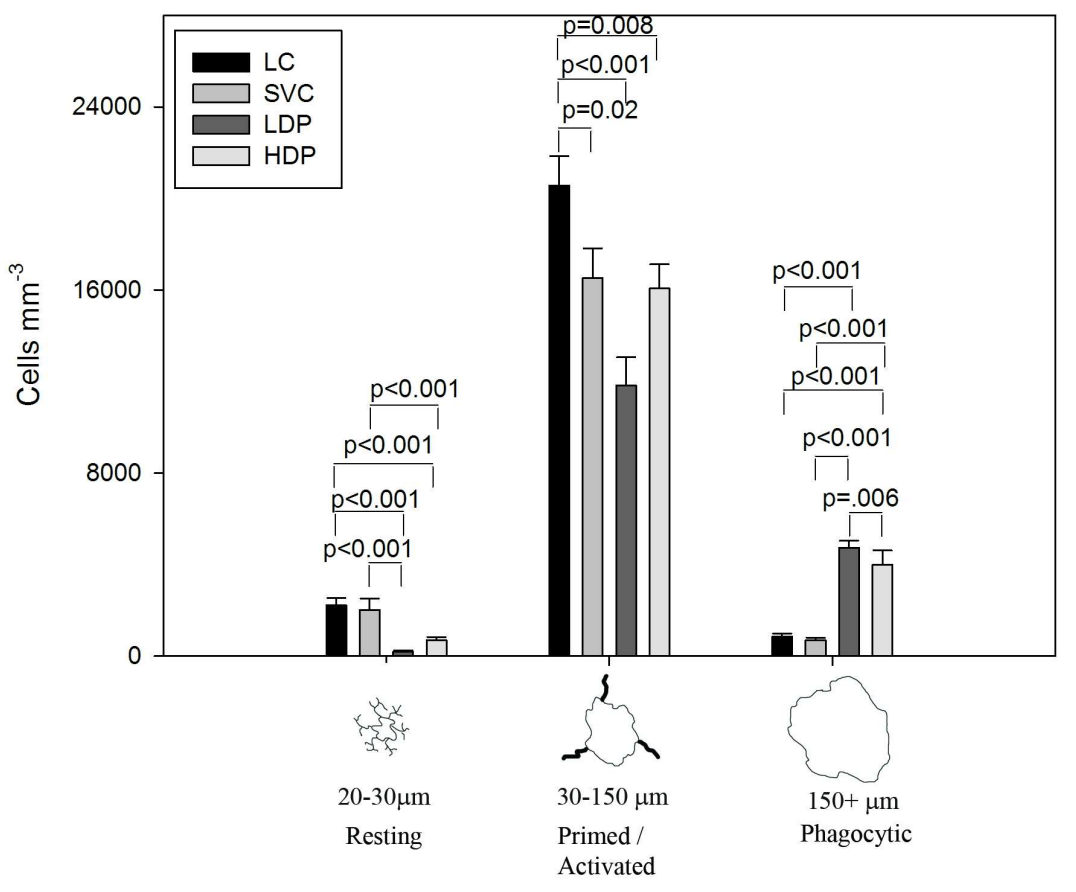

Figure 8 VEGF and PDGF delivered from an osmotic pump significantly effected the inflammatory cell population. The density of IBA1-positive cells classes surrounding the lesion at 1 month was significantly different between treatment and control groups. $182 \times 170 \mathrm{~mm}(300 \times 300 \mathrm{DPI})$ 

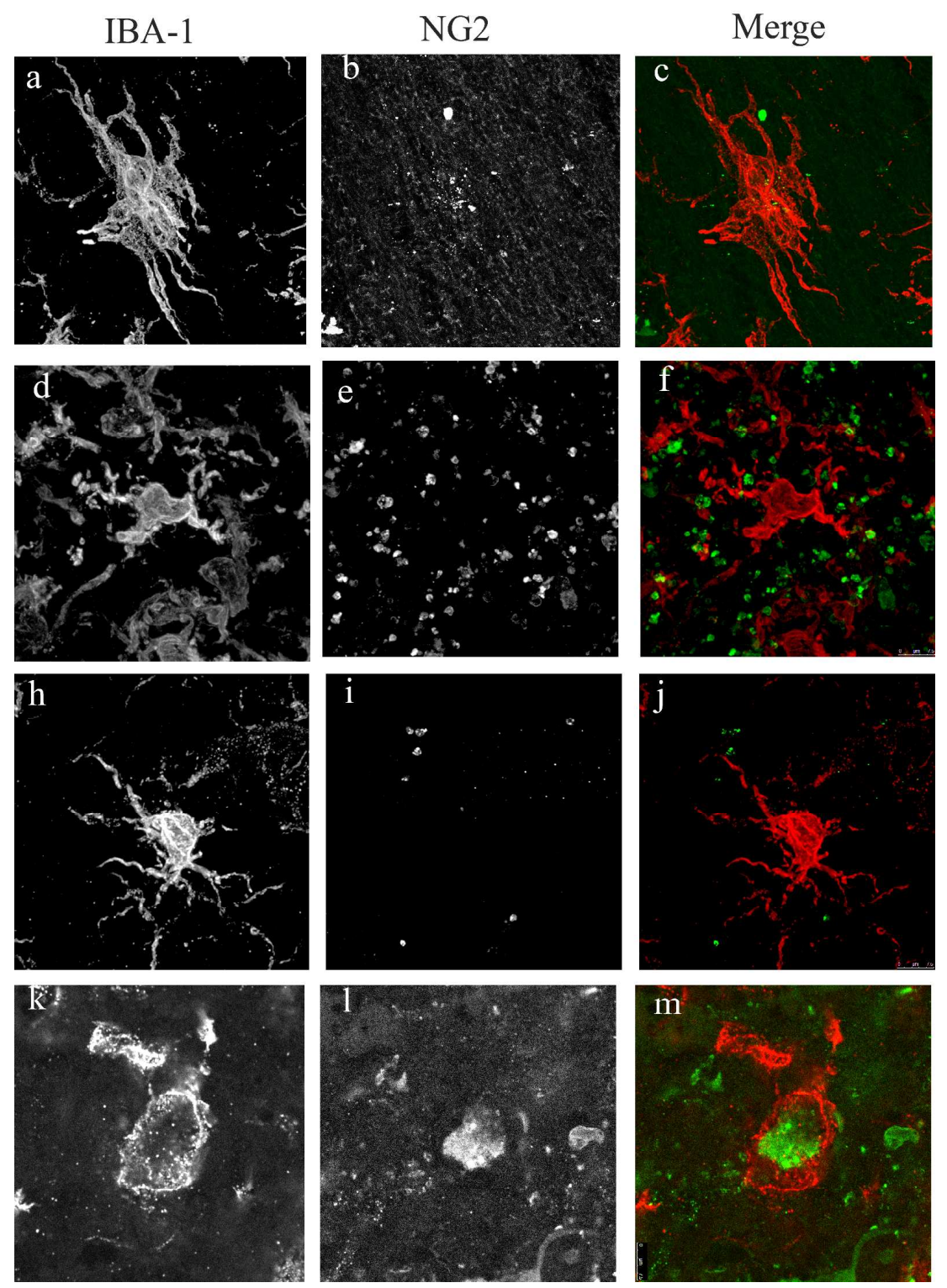

Figure 9 Confocal images of representative of IBA+ cells at 260x (a-c) lesion control (d-f) VEGF alone (h-j) PDGF alone (k-m) High dose VEGF/PDGF. (a,d,h,k) IBA (b,e,i,l) NG2 and $(c, f, j, m)$ merge. There are distinct differences in IBA morphology between the dual growth factor delivery and the other groups. NG2 staining is sparse in the lesion control and PDGF along groups, while there is isolated staining in the VEGF alone group and colocalisation with IBA in the dual growth factor treatment.

$269 \times 384 \mathrm{~mm}(300 \times 300$ DPI $)$ 